\title{
Autoconcepto y autocontrol en estudiantes del nivel secundario de instituciones privadas de Lima Sur
}

\author{
Selfconcept and self-control in students of secondary level of private institutions of \\ Lima sur
}

Yulian León Pacheco ${ }^{1 *}$, Raquel Peralta Vela ${ }^{1}$, Jania Jaimes Soncco ${ }^{2}$

\section{RESUMEN}

Objetivos: determinar si existe relación significativa entre autocontrol y autoconcepto en estudiantes del nivel secundario de instituciones privadas de Lima sur. Materiales y Métodos: Se utilizó el diseño no experimental, de corte transversal y de tipo correlacional. Asimismo, se empleó un muestreo no probabilístico intencional, incluyendo alumnos de ambos sexos con edades entre 11 y 18 años. Resultados: Los resultados mostraron que el $46.3 \%$ de los participantes presentan niveles bajos de autoconcepto, y el $71.1 \%$, presenta autocontrol en nivel promedio y alto. Además, existe una relación moderada positiva y altamente significativa entre nivel de autoconcepto global y autocontrol $(r=, 270$, $p<0.01$ ). Conclusiones: De acuerdo a ello, se concluye que el autoconcepto se relaciona moderada y significativamente con el autocontrol.

Palabras claves: Autocontrol, autoconcepto, adolescentes y estudiantes. (Fuente: DeCS BIREME)

\section{ABSTRACT}

Objetive: This research aimed to determine if there is a significant relationship between self-control and self-concept in students at the secondary level of private institutions in south Lima. Materials and Methods: Non-experimental cross-sectional and correlational design were used. Likewise, intentional non-probabilistic sampling was used between male and female students aged between 11 and 18 years. Results: The results showed that $46.3 \%$ of participants had low levels of self-concept, and $71.1 \%$ had self-control at medium and high levels. In addition, there is a moderate positive and highly significant relationship between the level of global self-concept and self-control $(r=, 270, p<0.01)$. Accordingly. Conclusions: We conclude that self-concept is related to moderate and significant selfcontrol.

Keywords: Self-control, self-concept, adolescents and students. (Source: MeSH NLM)

\footnotetext{
${ }^{1}$ Licenciado en Psicología. Consultoría privada, Lima, Perú.

2Docente de Psicología, Facultad de Ciencias de la Salud, Universidad Peruana Unión, Lima, Perú.
} 


\section{INTRODUCCIÓN}

En los últimos años, la ocurrencia de actos violentos en edades tempranas se ha incrementado. Con respecto a ello, la Organización Mundial de la Salud (OMS) (2015) y las Naciones Unidas, han reportado más de 200000 casos de suicidio cada año. El suicidio es una problemática compleja que está relacionada a trastornos mentales como la depresión y los trastornos por consumo de alcohol en Europa y América del Norte. Sin embargo, la conducta impulsiva es un importante factor de riesgo de suicidio. Así también, el Fondo de las Naciones Unidas para la Infancia (Unicef) (2014) muestra que, a nivel mundial, los adolescentes de 13 y 15 años de edad participaron en al menos una pelea. Del mismo modo, Povedano, Estévez, Martínez y Monreal (2012), manifiestan que gran parte de los adolescentes españoles carecen de estrategias para el autocontrol, y afirman que, debido a ello, el $16 \%$ de los estudiantes de 10 a 16 años de edad se han visto envuelto en casos de violencia escolar, teniendo en cuenta que el $7 \%$ de estos adolescentes han sido víctimas de acoso por sus iguales, el $7 \%$ se caracteriza por amenazar, intimidar y agredir a sus compañeros y el $2 \%$ muestran conductas agresivas. Del mismo modo, Fleitas y Otamendi (2012) mencionan que, en Argentina, un 42,3\% de los jóvenes ha vivido situaciones de violencia en el ámbito escolar entre compañeros.

En Perú, la Secretaría Nacional de la Juventud (SENAJU, 2015), muestra que el impacto que tienen la violencia y las adicciones, comprendidas como conductas carentes de autocontrol e impulsividad, se presentan principalmente en los jóvenes. Dicha encuesta, señaló también, que los principales problemas que afectan en un $58,6 \%$ a los jóvenes son la delincuencia y el pandillaje y el consumo excesivo de alcohol o drogas en un $39,5 \%$.

Una de las principales causas de agresión en adolescentes es la falta de autocontrol (Goleman, 1997), este es definido como un componente de la inteligencia emocional (Comas, Moreno y Moreno, 2003). Del mismo modo, la Real Academica Española (RAE, 2014), lo define como la capacidad que posee un individuo para poder regular y controlar sus impulsos.

En este sentido, Skinner, refiere que para desarrollar el autocontrol es necesaria la formación de otros componentes de la autoestima, como el autoconocimiento y autoconcepto; este último tiene una relación estrecha con el autocontrol, ya que es la variable que influye en el adecuado desenvolvimiento del aspecto social del individuo, lo cual se verá reflejado en su conducta y en el control que posee sobre ella (Rodríguez, 2007). Es por ello que, el autoconcepto, que es definido como la percepción que el individuo posee de sí mismo, es uno de los factores esenciales en el desarrollo de la personalidad de un individuo, ya que se encuentra relacionado con el bienestar general y un adecuado ajuste psicosocial (Shavelson, Hubner y Stanton, 1976).

La relación entre autoconcepto y autocontrol, ha sido estudiada por autores como Fernandes, Marín y Urquijo (2010), quienes realizaron un estudio correlacional de las variables mencionadas, en adolescentes de una escuela pública en Brasil, haciendo uso de la Escala Femenina y Masculina de Autocontrol y la escala de Autoconcepto Infanto-Juvenil, en el cual los resultados demostraron correlaciones medias.

Sin embargo, a nivel nacional e internacional existen pocos estudios que involucren estas variables; debido a ello, el objetivo de esta investigación es determinar si existe relación significativa entre autoconcepto y autocontrol, lo que permitirá obtener resultados específicos y una visión más concreta de la problemática.

\section{MATERIAL Y MÉTODOS}

Para llevar a cabo la investigación se utilizó el diseño no experimental de corte transversal y de tipo correlacional, siendo que la naturaleza de la investigación es relacionar las variables de estudio (Hernández, Fernández y Baptista, 2010).

\section{Participantes}

Participaron 320 adolescentes de entre 11 y 18 años de edad de ambos sexos, que cursaban el primero, tercero y quinto año de nivel secundaria en el año académico 2016, contando con la participación de tres instituciones educativas privadas de Lima sur, quienes completaron los cuestionarios de forma voluntaria, a través de un muestreo no probabilístico intencional. La muestra estuvo conformada por 140 mujeres $(43.8 \%)$ y 180 varones (56.3\%). El $62.8 \%$ se encontraba en la primera etapa de la adolescencia (11- 14 años). El 39.1\% cursaba el 
primer grado de secundaria. El $92.8 \%$ procedía de la costa y el $62.8 \%$ profesa la fe adventista.

\section{Instrumentos}

Los componentes del cuestionario incluyeron una declaración de consentimiento informado, el Test de autoconcepto Forma 5 y el Cuestionario de Autocontrol Infantil y Adolescente (CACIA).

\section{Test de autoconcepto Forma 5}

Esta escala fue creada en el año 2001 por García y Musitu en Madrid (España). Consta de 30 ítems tipo Likert divididos en 5 factores, que representan diferentes dimensiones:

Autoconcepto Académico (6 ítems), Social (6 ítems), Emocional (6 ítems), Familiar (6 ítems) y el Físico (6 ítems). Con una escala de respuestas donde se puntúa de forma directa colocando "1" si nunca sucede, con "2" si sucede casi nunca, con " 3 " si sucede algunas veces, con "4" si sucede casi siempre y con " 5 " si sucede siempre. La suma de las puntuaciones de los cinco factores, permite obtener la puntuación directa del autoconcepto total.

Portilla (2011) realizó un análisis psicométrico en Perú. Al establecer la validez de contenido a través del criterio de jueces, los resultados indicaron que los ítems del cuestionario AF5 puntuaron el valor mínimo de 0.80 , por lo que se afirma que este cuestionario posee una adecuada validez de contenido. En cuanto al análisis de confiabilidad, la consistencia interna, dio como resultado un coeficiente alfa de Cronbach de 0.872 , lo que indica que las puntuaciones de este cuestionario son confiables. Del mismo modo, muestra que las correlaciones ítem - test fluctúan entre 0.204 y 0.634 , sobrepasando el criterio de 0.20 , lo que permite afirmar que los ítems del cuestionario son consistentes.

\section{Cuestionario de Autocontrol Infantil y Adolescente (CACIA)}

Creado por Capafóns y Silva (2001), cuenta con escalas de autocalificación en tres dimensiones de autocontrol positivo (Retroalimentación Personal, Retraso de la recompensa y Autocontrol Criterial), una de autocontrol negativo (Autocontrol Procesual), y una escala de sinceridad. EI CACIA consta de 89 reactivos dicotómicos y su aplicación se dirige a niños y adolescentes entre 11 a 19 años, su administración puede ser individual o colectiva y tiene una duración entre 30 y 40 minutos. Su tipificación es a través de centiles para cada escala según ciclo escolar y sexo.

Rodriguez y Carretto (2007) realizaron el análisis de validez y fiabilidad del cuestionario en Perú. En primer lugar, elaboraron la validez de contenido, a través de criterios de jueces para lo cual se tomaron en cuenta a 10 expertos en el área y en metodología de la investigación. Para la confiabilidad realizaron un estudio referido al análisis psicométrico; en este análisis se encontró, según las escalas originales, que los coeficientes Alfa de las escalas retraso de la recompensa (Alfa de Cronbach punto .659) y autocontrol criterial (Alfa de cronbach .226) se encuentra por debajo de lo esperado, mientras que retroalimentación personal (Alfa de Cronbach punto .784), autocontrol procesual (Alfa de Crombach .711), por último sinceridad (Alfa de Crombach puntos .705), si cumplen con dicho criterio (por encima de puntos .70) se demostró que las 4 escalas de la prueba son confiables, por lo que se tuvo que eliminar la escala autocontrol criterial y además algunos ítems de otras escalas porque no cumplían con el valor psicométrico esperado. Finalmente, se realizó un análisis con los 71 ítems con la escala revisada, donde se observó que en todos los casos los valores de la correlación ítem-test corregida obtienen coeficientes mayores a .20 y un Alfa de Cronbach por encima de .70 (Kleine, 1993), lo que indica que el cuestionario adaptado que evalúo el autocontrol infantil y adolescente, es un instrumento confiable.

\section{RESULTADOS}

En la Tabla 1 se muestra que el $46.3 \%$ de los participantes presentan niveles bajos de autoconcepto. Además, se observa que el $53.7 \%$ de los estudiantes tienen un nivel de autoconcepto moderado y alto. De manera similar, se observan porcentajes entre promedio $y$ alto en el autoconcepto académico, social, familiar y físico. Finalmente, se evidencia que el $59.1 \%$ de los participantes presenta niveles bajos de autoconcepto emocional. 
Tabla 1

Niveles de autoconcepto en estudiantes del nivel secundario.

\begin{tabular}{lllllll} 
& \multicolumn{2}{c}{ Bajo } & \multicolumn{3}{c}{ Promedio } & Alto \\
\cline { 2 - 7 } & N & $\%$ & N & $\%$ & N & $\%$ \\
\hline Autoconcepto & 148 & 46.3 & 83 & 25.9 & 89 & 27.8 \\
Académico & 113 & 35.3 & 96 & 30.0 & 111 & 34.7 \\
Social & 153 & 47.8 & 105 & 32.8 & 62 & 19.4 \\
Emocional & 189 & 59.1 & 76 & 23.8 & 55 & 17.2 \\
Familiar & 152 & 47.5 & 91 & 28.4 & 77 & 24.1 \\
Físico & 128 & 40 & 104 & 32.5 & 88 & 27.5 \\
\hline
\end{tabular}

En la Tabla 2 se observa que el $71.1 \%$ de los participantes presenta autocontrol en nivel promedio y alto. Sin embargo, se evidencia que el $25.9 \%$ de la población posee bajo autocontrol. Por otro lado, se observan porcentajes altos en los niveles promedio y alto de autocontrol criterial y sinceridad. Finalmente, el $49.40 \%$ se ubica en la categoría bajo de la dimensión de retraso de la recompensa.

Tabla 2

Niveles de Autocontrol en estudiantes del nivel secundario.

\begin{tabular}{lllllll} 
& \multicolumn{2}{c}{ Bajo } & \multicolumn{3}{c}{ Promedio } & Alto \\
\cline { 2 - 7 } & $\mathrm{N}$ & $\%$ & $\mathrm{~N}$ & $\%$ & $\mathrm{~N}$ & $\%$ \\
$\begin{array}{l}\text { Autocontrol } \\
\begin{array}{l}\text { Retroalimentación } \\
\text { Personal }\end{array}\end{array}$ & 86 & 26.9 & 163 & 50.9 & 71 & 22.2 \\
$\begin{array}{l}\text { Retraso de la } \\
\text { Recompensa }\end{array}$ & 158 & 28.4 & 103 & 32.2 & 126 & 39.4 \\
$\begin{array}{l}\text { Autocontrol } \\
\text { Criterial }\end{array}$ & 78 & 24.4 & 177 & 55.3 & 65 & 20.3 \\
\begin{tabular}{l} 
Sinceridad \\
\hline
\end{tabular} & 65 & 20.3 & 226 & 70.6 & 29 & 9.1 \\
\hline
\end{tabular}

En la Tabla 3 se aprecia el análisis de correlación con la prueba estadística Rho de Spearman en la cual se observa que existe una relación moderada positiva y altamente significativa entre nivel de autoconcepto global y autocontrol $(r=, 270, p<0.01)$

Tabla 3

Coeficiente de correlación entre autocontrol y autoconcepto.

\begin{tabular}{lll}
\multirow{2}{*}{ Autoconcepto } & Autocontrol & \\
\cline { 2 - 3 } & Rho & $\mathrm{p}$ \\
Autoconcepto global &, $475^{* *}$ & .000 \\
Académico &, $459^{* *}$ & .000 \\
Social & $.252^{* *}$ & .000 \\
Emocional &, $150^{* *}$ & .007 \\
Familiar & $.294^{* *}$ & .000 \\
Físico &, $349^{* *}$ & .000 \\
\hline
\end{tabular}

** La correlación es significativa al nivel 0.01 


\section{DISCUSIÓN}

En el estudio se examina la relación que existe entre las 5 dimensiones del autoconcepto y el autocontrol, debido a que los reportes de casos de conductas que denotan agresión en adolescentes son cada día más frecuentes. Esta problemática se encuentra relacionada con el control y manejo de las propias conductas, lo que se vincula con el desarrollo y formación adecuada del autoconcepto (Rodríguez, 2010). De acuerdo con

ello, se comprueba la primera hipótesis, según la cual, existe relación moderada y altamente significativa entre estas dos variables. Además, este resultado coincide con el estudio realizado por Garaigordobil, Cruz y Pérez (2003); es decir, los adolescentes que evidencian una adecuada percepción de si mimos, tendrán mayores habilidades para el control de sí mismos.

En cuanto a la dimensión social del autoconcepto, en relación con la segunda hipótesis de esta investigación, se evidencia una correlación débil y altamente significativa, es decir, no en todos los casos, los niveles de autocontrol serán moderados, si es adecuada la percepción y conocimiento que el individuo posee acerca de sus cualidades más importantes de sus relaciones interpersonales, las mismas que le facilitan iniciar, mantener o ampliar una red social y su desenvolvimiento dentro de la misma (García y Musitu, 1993)

Del mismo modo, se comprueba la tercera hipótesis, ya que la dimensión emocional posee relación débil y altamente significativa con el autocontrol. Lo cual sugiere que el adolescente que posee una adecuada capacidad de reconocimiento y control de sus propias emociones, así como de sus respuestas frente a situaciones específicas, que conducirán al individuo hacia un estado de madurez (Goñi, 2009); no necesariamente manifestará un adecuado control de sí mismo.

En cuanto a la dimensión familiar del autoconcepto, se comprueba la cuarta hipótesis que afirma la existencia de relación altamente significativa con la variable autocontrol. Este resultado coincide con el estudio realizado por Fernandes Sisto et al., (2010), quienes mencionan que el adolescente que obtenga puntuaciones bajas en el autoconcepto familiar se caracteriza por ser una persona poco dispuesta a relacionarse, mostrándose desconfiado y poco leal con los miembros de su familia, lo cual ocasiona dificultad para adecuarse a las normas y del hogar, manifestado mediante conductas impulsivas, agresivas y de rebeldía.

Finalmente, se observa que, la dimensión física del autoconcepto posee una relación moderada y altamente significativa con la variable autocontrol. Lo que confirma la última hipótesis de la investigación, es decir, cuanto mayor sea la percepción del adolescente en lo que concierne a su aspecto y condición física (García y Musitu, 1993); lo que implica elegancia y cuidado personal, así como a la práctica de algún deporte, alimentación y descanso equilibrado que favorezca la salud física (Esnaola et al., 2011); mayor será el nivel de autocontrol.

Se concluye que, debido a los escasos estudios que vinculen ambas variables, el estudio contribuye, a enriquecer los antecedentes para el estudio de ambas variables, además, se recomienda considerar, para posteriores investigaciones, el estudio en poblaciones diferentes $y$ con un mayor número de participantes, así como la posible relación del autocontrol con otras variables; como autoestima, inteligencia emocional, habilidades sociales, asertividad, etc.

Declaración de financiamiento y de conflictos de interés:

El estudio fue financiado por los autores, quienes declaran no tener conflictos de interés.

\section{Correspondencia}

Yulian León Pacheco. Dirección: Facultad de Ciencias de la Salud, Universidad Peruana Unión, Carretera central Km 19, Ñaña, Lima, Perú.

Correo electrónico: yulianleon@upeu.edu.pe 


\section{REFERENCIAS BIBLIOGRÁFICAS}

Capafóns, A. y Silva, F. (2001). Cuestionario de AutoControl Infantil y Adolescente-5aedición. Madrid: TEA Ediciones, S.A.

Castañeda, A. (2013). Autoestima, Claridad De Autoconcepto Y Salud Mental En Adolescentes De Lima Metropolitana. Recuperado de: http://tesis.pucp.edu.pe:8080/repositorio/ handle/123456789/5094.

Rodriguez, M. y Carretto, L. (2007). Autocontrol: un estudio exploratorio.

Comas, R., Moreno, G.y Moreno, J. (2003). Aprendizaje Y Desarrollo. Consejo Mexicano de Investigación Educativa, AC,. Madrid: JUMA.

Esnaola, I., Rodríguez, A. y Goñi, E. (2011). Propiedades psicométricas del cuestionario de autoconcepto AF5. Anales de Psicologia, 27(1), $109-117$.

Fernandes, F., Marín, F. y Urquijo, S. (2010). Relación Entre Los Constructos Autocontrol Y Autoconcepto En Niños Y Jóvenes. Liberabit. Revista de Psicología, 16(2), 217-226.

Fleitas, D. y Otamendi, A. (2012). Mapa de la Violencia de Género en Argentina. Buenos Aires. Recuperado de: www.app.org.ar

Garaigordobil, M., Cruz, S. y Pérez, J.(2003). Análisis correlacional y predictivo del autoconcepto con otros factores conductuales, cognitivos y emocionales de la personalidad durante la adolescencia.

García, F. y Musitu, G. (1993). Rendimiento académico y autoestima en el ciclo superior de EGB. Revista de Psicología de La Educación, 4(11), 73-87. Recuperado de: https://www.uv.es/ lisis/gonzalo/16revista-psicol.pdf.

Goleman, D. (1997). La salud emocional (1ra ed.). Barcelona: Editorial Kairós. Recuperado de:https://books.google.es/books?hl=es\&lr=\&id=U_ OSZzeNMWcC\&oi=fnd\&pg=PA13\&dq=goleman+1997\&ots=ZmnqTXt3UV\&sig=DzJdQ8PxuFFAy0aDkTSceC_bXbl\#v=onepage\&q=goleman $1997 \& f=$ false.
Goñi, A., Esnaola, I. y Azua, S.(2003). Autoconcepto físico y desarrollo personal: perspectivas de investigación. Revista de psicología 15(16), 7-62. Recuperado de: https://doi.org/10.1387/ RevPsicodidact.156.

Hernández, R., Fernández, C. y Baptista, P. (2010). Metodología de la investigación. Mexico: McGrawHill. Recuperado de: https://es.scribd.com/ doc/38757804/Metodologia-de-La-InvestigacionHernandez-Fernandez-Batista-4ta-Edicion.

Organización Mundial de la Salud. (2015). Violencia juvenil. Recuperado de: http://www.who.int/ mediacentre/factsheets/fs356/es/.

Real Academia Española. (2014). Diccionario de la Lengua Española. Diccionario de la Lengua Española. Recuperado de: https://doi. org/10.2307/313964.

SENAJU. (2015). Informe Nacional de las Juventudes en el Perú. Perú. Recuperado de: http://www. unfpa.org.pe/publicaciones/publicacionesperu/ SENAJU-Informe-Nacional-Juventudes-2015. pdf.

Shavelson, R., Hubner, J. y Stanton, G.(1976). Self-Concept: Validation of Construct Interpretations. Review of Educational Research, 46(3), 407-441. Recuperado de: https://doi. org/10.3102/00346543046003407.

Unicef. (2014). Informe anual de femicidio 2014.

Recibido: $18 / 04 / 17$

Aceptado: 19/09/17 